Research Article

Gafar G. Ismayilov, Vugar M. Fataliyev*, and Elman Kh. Iskenderov

\title{
Investigating the impact of dissolved natural gas on the flow characteristics of multicomponent fluid in pipelines
}

https://doi.org/10.1515/phys-2019-0021

Received Aug 16, 2018; accepted Mar 07, 2019

Abstract: The conventional equations for describing the flow characteristics of the mixtures merely consider fluid that is homogenic, if it is above the bubble point conditions but ignore that a system containing sub-micron sized gas or vapor bubbles distributed throughout the volume of the liquid, which can exhibit unexpected heterogenic and complex phase properties. In this paper, a new mathematical model for the flowing gas-liquid mixture is presented, which has been proposed considering the colloidal feature of the system above the saturation or bubble point pressure. This approach is more in line with the actual dynamic performance of the oil and gas mixture export pipeline.

Experimental data, simulations and field case studies validate the new proposed mathematical model of flow characteristics in pipeline. The obtained results confirmed that the calculated data are in good agreement with the experimental data. Based on Azerbaijan oil-gas-condensate field "Guneshli" data, this new model was used for calculating the condition in which the transformation of the flow characteristics from stable into instable is occurred. It has been discovered that the flow becomes unstable at a pressure about 30\% higher than Bubble Point Pressure, which causes pulsation effect in the pipeline structure. However, homogenic behavior should be observed in this hydrodynamic condition. Also, the model provides a guideline on how to optimize the flow rate by adjusting the pipeline parameters to minimize the flow resistance, liquid slugging and hydraulic hammering effects, which cause instable operation.

\footnotetext{
${ }^{\star}$ Corresponding Author: Vugar M. Fataliyev: Azerbaijan National Academy of Sciences, Institute Geology and Geophysics, Azerbaijan; Email: fatavm@bp.com

Gafar G. Ismayilov: Azerbaijan State Oil and Industry University, FacultyGas, Petroleum and Mining, Azerbaijan

Elman Kh. Iskenderov: Azerbaijan State Oil and Industry University, Department Oil and Gas Transportation and Storage, Azerbaijan
}

əopen Access. @ 2019 G. G. Ismayilov et al., published by De Gruyter. (cc) BY 4.0 License
Keywords: Gas-liquid mixture; Flow characteristics; Multicomponent Systems; Pipeline parameters; Mathematical model

PACS: 47.20.Hw, 47.27.nf, 47.55.-t, 47.55.Ca

\section{Introduction}

Multiphase flow is found in many places of petroleum industry. It occurs in the reservoirs, oil and gas wells, fluid export systems, refineries and petrochemical activities [6, 7, 17]. Predicting reservoir and pipe or flowlines performance and economy requires accurate modelling of the hydrodynamics of this process [2, 4, 11]. Mathematical modelling is commonly used for resolving this flow characteristics and associated issues $[8,28,29]$. However, it is quite complex process and difficult to develop a reliable model for predicting the occurrences correctly $[19,30]$. That is reason, experimental determinations and empirical correlations are applied to improve the reliability of the thermodynamic and hydrodynamic aspects of the mathematical models [3, 9, 23].

If phase transformation happens during exporting the multicomponent mixture, it causes instable-slugging operation due to flowing different type of medium at the same cross section of the pipe. Many negative consequences related to the severe slugging flow exist there. Thus, lots of investments and effort have been put into reducing or eliminating the severe slug $[15,20]$. The paper $[20]$ reviews in details the state-of-the-art related to analysis, detection, dynamical modeling and elimination of the slug within the offshore oil and gas exploration and production processes. The effects of empirical correlations of friction factors on the numerical stability and parameters predictions in stratified gas-liquid pipelines flow was studied using the two-fluid modeling the paper [10], where unequal phase pressure effects were considered. The main objective of the study [25] was to achieve a comprehensive integrated two-phase/single-phase hydrodynamic model for 
gas-condensate flows through transmission pipelines under industrial operating conditions. For liquid film holdup, Grolman and Fortuin model and Taitel and Duckler approaches were employed for uphill and downhill twophase flows, respectively. Then 160 different field data were applied to make the required corrections to the model including the development of two new correlations for both liquid-gas and liquid-wall friction factors.

A slow transient was simulated using Godunov numerical scheme to solve the model equations in the paper [26]. The results proved that including the inertia term in the momentum equation makes the model more realistic. In the paper [5] a slug flow load model was proposed to simulate the oscillations induced by the internal flow on pipelines conveying liquid and gas mixtures. The internal flow loads imposed on the pipelines were deduced and implemented in a 3D simulator of pipelines structural dynamics. In order to evaluate the resonance effect caused by internal two-phase flow, a dynamic model capable of describing axial, bending vibrations and fluid-structure interaction was formulated using finite element method, where the void fractions of the two-phase flow were predicted by empirical correlations [27].

Generally, the analysis of the present-day studies on the flow of the multicomponent systems in the pipelines show that most of them examined the processes occur in regions below bubble point (BPP) or dew point pressure (DPP), because phase transformation takes place in this zone. In accordance with these papers the multicomponent mixtures above or close to the BPP/DPP were considered as the homogeneous media, as usually the system is still identified in a single-phase condition $[2,18]$. However, some of the studies $[1,12]$ have shown that the multicomponent systems can display structural inhomogeneity due to the formation of micro-embryos of liquid phase in gas medium or sub-micron sized vapor bubbles in the liquid phase when it is close to the phase transformation state. The paper [24] presents the experimental studies of the phase behavior and the viscosity of the oil at different pressures. It was observed that the viscosity rapidly decreased at the pressure range 1-1.14 times above the BPP. In the papers $[13,22]$ it is found that a system containing gas or vapor sub-micron sized bubbles distributed throughout the volume of the liquid, can exhibit non-equilibrium phase properties. This circumstance can be expected to contribute to significant changes in hydraulic characteristics and trigger unstable operation, which causes various complications and additional costs when transporting gasliquid mixture.

This literature survey concluded fundamental findings, which should be considered for further learning and understanding the occurrences in pipeline, when flowing complex system is in above the BPP condition. Considering the given details, in this paper, an attempt has been made to concept an effective mathematical model for the flow of multicomponent or gas-saturated systems in a pipeline in the vicinity and presence of phase transformations.

\section{Fundamentals of multicomponent hydrocarbon systems and some important aspects of flow characteristics in the pipeline}

\subsection{About the features of the phase envelope for multicomponent hydrocarbon system}

Natural hydrocarbon fluids contain many components therefore, phase-composition data can no longer be represented with multi coordinates. Instead, phase diagrams that give more limited information are used. Figure 1 illustratively shows a pressure-temperature phase diagram for a multicomponent mixture which can be representative of systems flowing in the pipelines.

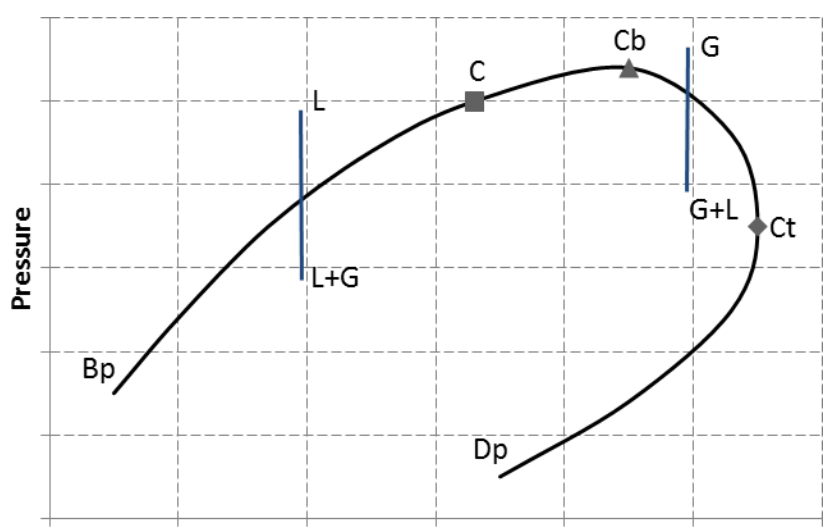

Temperature

Figure 1: Pressure-temperature phase envelope for multicomponent mixture

In general, the phase diagram consists of two sections which are left and right-hand sides of system critical point (C). (C) is the temperature and pressure at which the phase compositions and all phase properties are identical. (CDp) line holds dew point pressures (DPP) and temperatures and (CBp) line presents the gas saturated pressure 
(BPP) and temperatures. At temperatures greater than the cricondentherm $(\mathrm{Ct})$, which is the maximum temperature for the mixture of two phases, only one phase occurs at any pressure. At pressure greater than the cricondenbar $(\mathrm{Cb})$, which is the maximum pressure for the mixture of two phases, only one phase occurs at any temperature, but it could be liquid or gas depending on whether the temperature is below or above the critical temperature. Figure 1 shows the phase change of a multicomponent system in which the temperature is between the critical temperature and the cricondentherm, and the pressure is larger than the DPP. During the flow in pipeline, the temperature can remain constant. As the pipeline pressure declines, shown as vertical line $\mathrm{G}(\mathrm{G}+\mathrm{L})$ in Figure 1, it goes through the upper dew point from point $\mathrm{G}$ (gas) at the pipeline temperature and liquid begins to precipitate out of the gas phase.Decreasing pressure causes liquid to appear in the pipeline when the DPP is reached. As the pressure declines further, the saturation of liquid increases (point $\mathrm{G}+\mathrm{L}$ ) because of retrograde condensation.

Also, Figure 1 shows the phase change of a multicomponent system in which the temperature is below the critical point, and the pressure is larger than the BPP. As the pipeline pressure declines in isothermal condition, shown as vertical line $L(L+G)$ in Figure 1, it goes through the bubble point from point $\mathrm{L}$ (liquid) at the pipeline temperature and gas begins to separate out of the liquid phase. Decreasing pressure causes gas to appear in the pipeline when the BPP is reached. As the pressure declines further, the twophase flow occurs because of liquid and gas separation (point $\mathrm{L}+\mathrm{G}$ ). This condition is sometimes called undersaturated because the fraction of light components present in the liquid is low for a gas phase to form at that temperature and pressure. The appearance of the much more compressible vapor phase reduces the rate of pressure decline. The volume of vapor present in the pipeline grows rapidly with reduction of pressure below the bubble point.

It is known that if any one of the leading thermodynamic parameters changes, the given system moves to the next state. If this change results in a phase transition, then at first, micro embryos of the new phase appear and then slowly grow according to the leading parameter. Microscopic particles of one phase dispersed in another are generally called colloidal solutions or dispersions [21]. Multicomponent hydrocarbon systems are also known as colloidal dispersions when it's close to the phase transition state because some components remain in dispersed condition however, thermodynamic and hydrodynamic features of the mixture significantly change [1]. A common method of classifying colloids is based on the phase of the dispersed substance and in what phase it is dispersed. The types of colloids include sol, emulsion, foam, and aerosol [21]. Aerosol contains small particles of liquid or solid dispersed in a gas. In multicomponent hydrocarbon systems, it happens when pressure is close to the retrograde condensation or upper DPP (see Figure 1, line- $\mathrm{G}(\mathrm{G}+\mathrm{L})$ ). Foam is formed when many gas particles are trapped in a liquid or solid. For natural hydrocarbon mixture, it happens when the system is near the BPP (see Figure 1, line-L(L+G)).

\subsection{Phase envelope for multicomponent hydrocarbon system flowing in the pipeline}

It is assumed that the gas saturated liquid is flowing in the pipe (see Figure 2) with a diameter $(d)$, and with length $(l)$. The gas ratio of the flowing fluid (dissolved gas per liquid volume) is $(\beta)$. The pressure at the inlet much higher than the formation of gas phase embryos or micro sized bubbles pressure $\left(P_{m s b}\right)$ but the pressure is on other end of pipe $\left(P_{\text {out }}\right)$ or at the outlet is below the BPP or saturation pressure $\left(P_{K}\right)$.

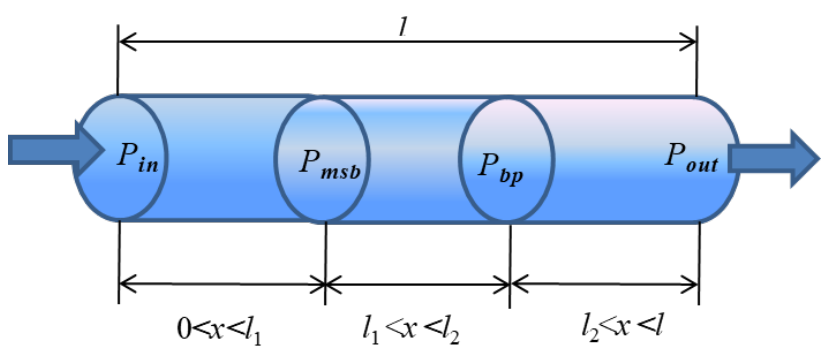

Figure 2: Different flow characteristics of multicomponent systems in the pipeline

In order to assess the hydraulic and hydrodynamic characteristics of the fluid along the length of the pipeline, taking into account the phase transformations, three zones or pipe sections can be distinguished (Figure 2). In the first zone $\left(0<x<l_{1}\right)$ where $P_{i n}>P_{m s b}$, the flow is considered homogeneous. It is assumed that at the end of this section the pressure of the $P_{m s b}$ is reached or microembryos of the gas phase (micro sized bubbles) begin to appear, and these tiny bubbles start influencing on flow features. Then, the concentration of the gas bubbles begins to grow intensively with a further drop of pressure in the second section. For this section $\left(l_{1}<x<l_{2}\right)$ of the pipeline, it can be accepted that the viscosity of the system is a function of gas concentration and can be expressed accordance to A. Einstein equation. As a final point, the 
third section of the pipeline is assumed to be a region of a two-phase flow, in which $\left(l_{2}<x<l\right)$ according to the generally accepted judgment, the system starts moving to two-phase flow regime as the pressure drops to BPP $\left(P_{b p}\right)$ level $\left(P=P_{b p}\right)$ at the point of $l_{2}$. Free gas separation from the liquid occurs and depending on the thermodynamic conditions the variation of gas-liquid flow structure could take place.

As it is seen the unique phase behavior of the natural oil and gas mixture typically causes a nonequilibrium thermodynamic two-phase flow in the transmission pipeline, in which the liquid and vapor phases have different characteristics at the same cross section of the pipe. In the paper [14], to describe this two-phase flow, a one-dimensional two-fluid model considering the fluid compressibility and viscosity was built based on the general mass, momentum and energy conservation equations for each phase. To select its appropriate solution method in the work [14], the mathematical characteristic of the model was studied using the eigenvalue analysis method. The results demonstrated that its mathematical characteristic is primarily dependent on the void fraction, densities and flow velocities of the liquid and vapor phases. The two-fluid model is hyperbolic and well-posed when the liquid flow velocity is equal to the vapor flow velocity, the two-phase flow reduces to a single-phase flow, or the difference between the liquid velocity and the vapor velocity is greater than the mixture sound speed. Otherwise, the model is non-hyperbolic and ill-posed. Additionally, the results indicated that the numerical solution methods of the adiabatic two-fluid model could be potentially extended to solve the non-equilibrium two-fluid model for the natural gas liquid pipe flow. This approach solves and describes some of the hydrodynamic aspects of the flow, but the situation changes significantly if flowing multicomponent system contains light gas components solved in the heavy hydrocarbons. In this case, difficult to identify the critical points where single-phase transfers into multiphase state. Our approach below is different as we try to describe the occurrences in the flowing multicomponent fluid in the pipeline if dissolved gas components exist in the system in micro sized bubbles condition.

\section{Mathematical modeling the flow of multicomponent system including the condition is close to bubble point}

\subsection{Mathematical model for the flow of gas-saturated systems in a pipe in the presence of phase transformations}

Based on the explanation given in the sections above, the total pressure lose for the pipeline can be expressed as Eq. (1). This equation was originally given in the paper [16] but it was carefully investigated and improved significantly by Ismayilov in the work [13]. In accordance with this work the model is simple and reliable for practical calculations, which are the main reasons for choosing this equation.

$$
\begin{aligned}
& \Delta P=\lambda \frac{l_{1}}{d} \frac{W^{2}}{2 \rho^{\prime}}+\lambda(1+\gamma c) \frac{l_{2}-l_{1}}{d} \frac{W^{2}}{2 \rho^{\prime}}+\lambda \frac{l-l_{2}}{d} \frac{W^{2}}{2 \rho^{\prime}} 1 \\
& +\phi \bar{x}\left[1+\phi \bar{x}\left[\frac{\rho^{\prime}}{\rho^{\prime \prime}}-1\right]\right]
\end{aligned}
$$

where $\rho^{\prime}, \rho^{\prime \prime}$ are the densities of the liquid and gas phases respectively, $W$ is the mass flow rate, $\phi$ is the two-phase flow parameter, $\bar{x}$ is the average mass gas content, $\lambda$ is the hydraulic resistance coefficient, $c$ is the gas concentration, $\gamma$ is a coefficient that depends on the size and shape of the gas phase embryos or bubbles in the system.

Taking into account the fact that when the pressure in the system decreases, the enthalpy $(h)$ of the gas-saturated stream changes by the quantity of $\frac{\partial h}{\partial P} \frac{\partial P}{\partial t}$. It can be detremined according to the paper [16] if mass flow rate $(W)$, the length of the first section of the pipe $\left(l_{1}\right)$ and no presence of gas bubbles in the system is known

$$
\frac{\pi d^{2}}{4} \rho^{\prime} l_{1} \frac{\partial h}{\partial P} \frac{\partial P}{\partial t}
$$

where $h_{m s}, h_{i n}$ the enthalpy of the flow at the pressures $\left(P_{m s b}\right)$ and $\left(P_{i n}\right)$ of the pipeline sections, respectively.

Identical approach can be applied to the other two sections of the pipeline. Considering the thermodynamic conditions, it can be expressed

$$
\begin{array}{r}
\frac{\pi d^{2}}{4} \rho^{\prime}\left(l 2-l_{1}\right) \frac{\partial h}{\partial P} \frac{\partial P}{\partial t}=\frac{\pi d^{2}}{4} W\left(h_{b p}-h_{m s b}\right) \\
\frac{\pi d^{2}}{4} \rho^{\prime}(1-\beta)\left(l-l_{2}\right) \frac{\partial h}{\partial P} \frac{\partial P}{\partial t}=\frac{\pi d^{2}}{4} W\left(h_{o u t}-h_{b p}\right)
\end{array}
$$

where $h_{b p}, h_{\text {out }}$ the enthalpy of the system at the BPP $\left(P_{b p}\right)$ and at the outlet pressure $\left(P_{\text {out }}\right)$ of the pipeline, respectively. 
Given that the gas content (mass) at the outlet of the pipeline can be represented as $\left(h_{\text {out }}-h_{b p} / r\right)$ (where $r$ is the degassing (separation) energy), then taking into account (4), for the average value of the mass gas content can be written

$$
\bar{x}=\frac{1}{2} x_{\text {out }}=\frac{\rho^{\prime}(1-\beta)\left(l-l_{2}\right) \partial h}{2 W r} \frac{\partial h}{\partial P} \frac{\partial P}{\partial t}
$$

Where, $x_{\text {out }}$ is the free (separated) gas content at the end of the pipe. Substituting the relations for $l_{1}, l_{2}$ and $\bar{x}$ from Eq. (2), (3) and (4) into Eq. (1) and expressing the mass velocity by using the volumetric flow rate of the system (Q) and after the simple transformations it can be obtained

$$
\Delta \mathrm{P}=\mathrm{AQ}^{3}+\mathrm{BQ}^{2}+\mathrm{CQ}
$$

where

$$
\begin{aligned}
& \mathrm{A}=\frac{16 \lambda \rho^{\prime}\left[\left(\rho^{\prime} / \rho^{\prime \prime}\right)-1\right]}{\pi^{3} d^{7} \frac{\partial h}{\partial P} \frac{\partial P}{\partial t}} \\
& \left.\left[\frac{2 \gamma c\left(h_{d p}-h_{m s b}\right)}{\left(\left(\rho^{\prime} / \rho^{\prime \prime}\right)-1\right)}+\frac{\phi(1-\beta)}{r}\left(h_{b p}-h_{\text {out }}\right)\right)^{2}\right] \\
& \mathrm{B}=\frac{8 \lambda l \rho^{\prime}}{\pi^{2} d^{5}}\left[1-\frac{\phi(1-\beta)}{r}\left[\left(\rho^{\prime} / \rho^{\prime \prime}\right)\right]\left(h_{b p}-h_{\text {out }}\right)\right] \\
& \mathrm{C}=\frac{\lambda \phi(1-\beta) \rho^{\prime}\left[\left(\rho^{\prime} / \rho^{\prime \prime}\right)-1\right] l^{2}}{\pi d^{3} r} \frac{\partial h}{\partial P} \frac{\partial P}{\partial t}
\end{aligned}
$$

This equation provided us with a greater understanding of the flow characteristics at the various sections of the pipelines which is going to be discussed in next sections.

\subsection{Results and discussions}

As it is seen from equation (6), the correlation between $\Delta \mathrm{P}$ and $\mathrm{Q}$ is expressed by the third-degree equation. An analysis of this model shows that the flow characteristic could be stable along the pipe length if the equation (6) had one real and two complex roots. Also, this model indicates that, the frictional pressure loss could theoretically be the same even at three different flow rates. In this case, this kind of an unstable flow characteristic should have two extremes and the flow rate of the system corresponding to that can be determined from equation below

$$
\frac{d \Delta P}{d Q}=3 \mathrm{AQ}^{2}+2 \mathrm{BQ}+\mathrm{C}=0
$$

Obviously, having the presence of a stable characteristic, Eq. (8) should not have real roots, which is possible if $\mathrm{B}^{2}<3 \mathrm{AC}$ has been determined from Eq. (7) taking into account that $h_{b p} \rightarrow h_{m s b}$. That means the second and the third sections of the pipeline (Figure 2) are combined and it is assumed that the phase transformation in the system occurs with the formation of the micro sized gas bubbles in the system. If one substitutes the relations for A, B and $C$ from the equation (7) into the condition $B^{2}<3 A C$ and carries out the simple transformations, one more agreement for the stability of the characteristic can be obtained, which is

$$
\frac{0,54\left[\left(l_{1} / l_{2}\right)-1\right]}{\left(\rho^{\prime} / \rho^{\prime \prime}\right)-1}<\phi x_{\text {out }}<\frac{7,46\left[\left(l / l_{1}\right)-1\right]}{\left(\rho^{\prime} / \rho^{\prime \prime}\right)-1}
$$

Based on these provided equations the criteria could be determined to control the flow in the pipelines and identify the conditions for optimizing the pressure loss during the exporting multicomponent hydrocarbon or liquid-gas mixed systems.

\subsection{Validation the reliability of provided mathematical model by experimental, simulation and field cases}

\subsubsection{Experimental validation and simulation case}

Special experimental module was constructed for investigation the hydrodynamic and thermodynamic characteristics of the flowing multicomponent system in the pipe and in different regime. This module consists of pipe with $5.6 \mathrm{~m}$ length and $0.016 \mathrm{~m}$ in diameter. The module is also, equipped with pump, gas compressors, temperature, flow and pressure controllers, oil reservoirs, gas accumulators and other instrument devises for precise control of processes and measurements. Beside that overpressure, overtemperature and overfilling protection devices were constructed for safe operations. As multicomponent system, crude oil and natural gas (field "Guneshli" in Azerbaijan) was used. These samples were collected from filed separator liquid and gas outlet lines accordingly. Experimental procedure and experimental scheme were described in [12]. Oil (viscosity: $0.022 \mathrm{~Pa} \times \mathrm{s}$; density: $866 \mathrm{~kg} / \mathrm{m}^{3}$ ) and natural gas (density: $0.7203 \mathrm{~kg} / \mathrm{m}^{3}$ )were mixed in the separate high pressure vessel at the inlet of the pipe under stable temperature and high pressure (1.5 times above DPP) ensuring singly phase state was achieved. All experiments were carried out in isothermal condition to eliminate temperature impact on the process.

During experiments the relationship of multicomponent system flow rate between sum differential pressure or pressure loss across the pipe length has been calculated. Calculations were carried out according to the Eq. (6) but for gas-liquid compositions and experimental data were referred to work [13, 22]. The obtained results confirmed that the calculated data is in good agreement with the experi- 
mental data (average tolerance has not exceeded 3\%). Using this data of the gas-liquid systems identified the values of the coefficients A, B, C (eq. (8)) as a function of the gas content which were presented in Figure 3. As seen from Figure 3 the stable and unstable zones of the flow characteristics can be easily recognized and also, it presents that the given system flow features can be forecasted using this model.

According to the Eq. (9) the regions of stability and instability of flow characteristics of gas-liquid systems were designed depending on the different values of the parameters $l_{1} / l_{2}$ and $\rho^{\prime} / \rho^{\prime \prime}$ and are shown in Figure 4.

As seen in Figure 4, the appearance of instability flow profile in the system can be observed in either conditions, at large or at sufficiently small amount of the mass content of the gas and in between them. In this case, if the condition (9) is met by adjusting the flow rate $(\mathrm{Q})$ or (complex

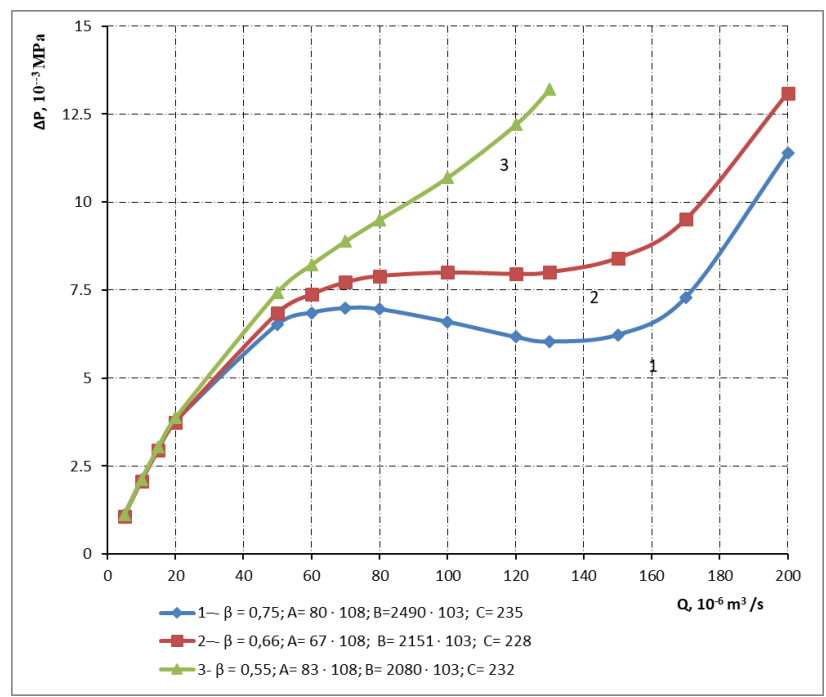

Figure 3: The flow characteristics of the flowing complex gas-liquid system base on given mathematical model

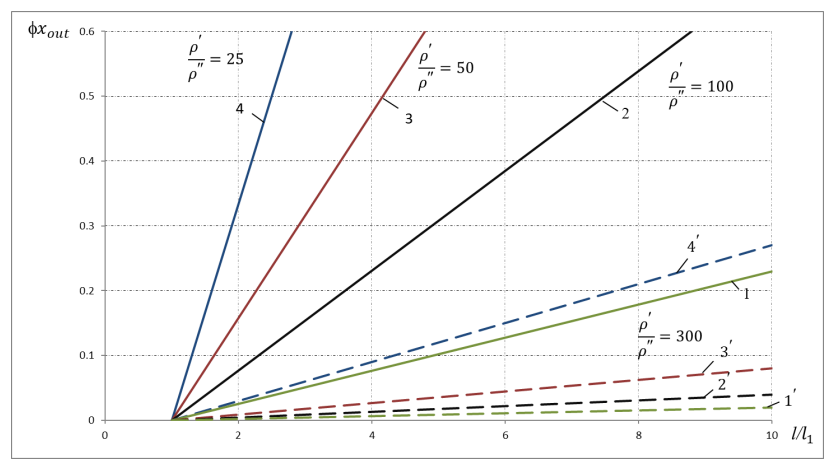

Figure 4: Boundary conditions for stable flow characteristics (1, 2, 3 and 4 lines indicate upper limits. 1', 2', 3' and 4' lines indicate lower limits) $\left.\phi x_{\text {out }}\right)$ and choosing $\left(l_{1} / l_{2}\right)$ in accordance with the pressure variation along the length of the pipeline, the instability of the hydraulic characteristics can be avoided.

The results of this research were obtained from theoretical and experimental investigations thus these findings require confirmation in real oil-gas export flow line conditions.

\subsubsection{Field case}

As oil and gas field development moves further into deep seas, maximizing hydrocarbon extraction at an acceptable cost and in safe operations mode are the greatest challenges facing the industry today. In this regard, considerable attention has been given to understanding the flow behavior in long and deep flow line risers of different topologies through transient multiphase simulation [2]. With this in mind, we used analysis of the performance of several oilgas export flow lines from Azerbaijan oil-gas-condensate field "Guneshli". This offshore field has been in operation since 1977. Deep Offshore Installations (DOI)- platforms were built to exploit multiple wells. Some of the highpressure wells directly (naturally) flowing to the export pipeline but low-pressure wells flow to the bulk separator for initial separation then oil is pumped to export.

As an example, in field case, oil and gas mixture export line between DOI-5 and DOI-2 analysis are presented in Figure 5. Statistics indicated that this pipeline expressed vibration, damaged pipe supports and brackets many times due to unexpected movements.

The reservoir fluid and pipeline properties for this case are presented in Table 1 and Figure 5. Based on this data,

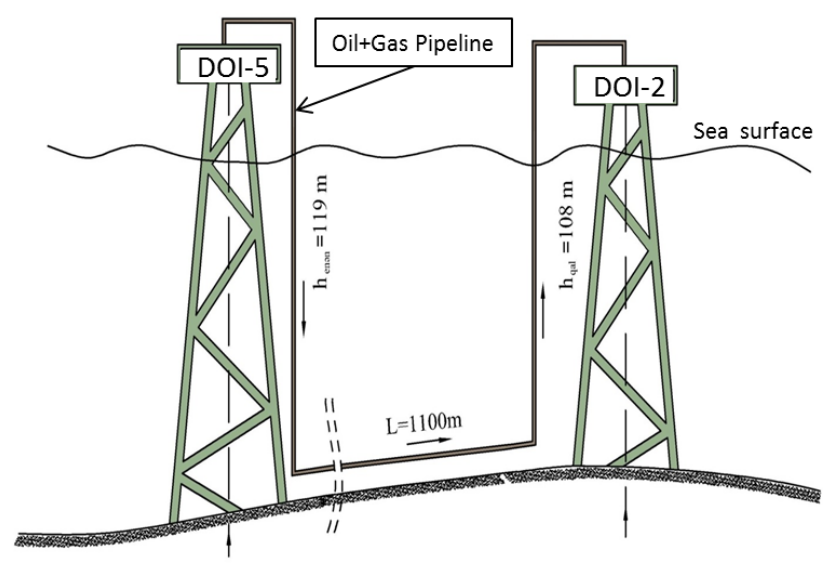

Figure 5: Flow characteristics of oil-gas mixture export pipeline from platform DOI-5 to DOI-2 on Guneshli oil-gas-condensate field (Azerbaijan) 
Table 1: Some additional information for field case

\begin{tabular}{lcccccccccc}
\hline Parameters & $\begin{array}{c}\text { Pipeline } \\
\text { diameter, } \mathrm{m}\end{array}$ & $\begin{array}{c}\text { Oil flow } \\
\text { rate, } \\
\mathrm{m}^{3} / \mathrm{s}\end{array}$ & $\begin{array}{c}\text { Gas flow } \\
\text { rate, } \\
\mathrm{m}^{3} / \mathrm{s}\end{array}$ & $\begin{array}{c}\text { Oil density, } \\
\mathrm{kg} / \mathrm{m}^{3}\end{array}$ & $\begin{array}{c}\text { Gas } \\
\text { density, } \\
\mathrm{kg} / \mathrm{m}^{3}\end{array}$ & $\begin{array}{c}\mathrm{T}, \\
\mathrm{K}\end{array}$ & $\begin{array}{c}\mathrm{P}_{\text {in }}, \\
\mathrm{MPa}\end{array}$ & $\begin{array}{c}\mathrm{P}_{03}, \\
\mathrm{MPa}\end{array}$ & $\begin{array}{c}\mathrm{P}_{H}, \\
\mathrm{MPa}\end{array}$ & $\begin{array}{c}\mathrm{P}_{\text {out }} \text {, } \\
\mathrm{MPa}\end{array}$ \\
\hline Values & 0.3 & 0.0153 & 10.254 & 865 & 0.857 & 283 & 1.65 & 1.27 & 0.96 & 0.81 \\
\hline
\end{tabular}

Eqs. (6) and (9) were used for calculating the conditions in which transformation from stable to instable flow characteristics occurred. It was discovered that the flow transit to unstable condition at a pressure about $30 \%$ higher than BPP caused pulsation and hammering effect. It means, if micro size gas bubbles appear in the system it significantly impacts on flow characteristics even laminar flow regime should be expected as per traditional calculations. The obtained results, also, helped to optimize the flow characteristic (see Figure 4) by adjusting the differential pressure. The flow resistance and pipeline vibrations reduced accordingly. Also, the pressure pulsation (amplitude) at the DOI-5 reduced from $0.57 \mathrm{MPa}$ to $0.23 \mathrm{MPa}$ and stabilised significantly.

Furthermore, the analyses and outcomes confirmed that the phenomenon (micro embryo condition of new phase) can cause unstable operation during exporting the hydrocarbon composition mixture resulting from a combination of different factors, including fluid phase properties, nature of compositions, thermobaric conditions, flow characteristicsand differential pressure between the export stations and pipeline parameters. If these factors are not analysed correctly at the beginning of field development and construction field, performance may suffer.

\section{Conclusion}

When the multicomponent mixture is in the region of saturation pressure and higher, the non-linear dependences and, in some cases, no monotonic appearances are observed on the flow characteristics. This circumstance can be expected to contribute to significant changes in thermodynamic and hydrodynamic characteristics, increasing differential pressure and triggering unstable operations which are often the cause of various complications and additional costs when transporting gas-liquid mixture. In this paper, a new mathematical model for the flowing gasliquid mixture has been proposed considering the highlighted aspects.

Presented new equation provides a greater understanding of the flow behaviour at the various sections of the pipeline. These pipeline sections were selected in order to assess the system flow characteristics when it is in purely liquid, foam (micro gas or vapour bubbles within the liquid) and two phases (free liquid and gas) conditions. According to this model, the phase transformation in the system occurs with the formation of the micro sized gas bubbles in the system. It was identified that, the correlation between sum pressure loss and flow rate across the pipeline can be expressed by the third-degree equation. An analysis of this model showed that the flow characteristics could be stable along the pipe length if the equation had one real and two complex roots only.

Experimental data, simulation and field cases studies validate the new proposed mathematical model of flow characteristics in pipeline. The obtained results confirmed that the calculated data are in good agreement with the experimental data. Based on Azerbaijan oil-gas-condensate field "Guneshli" data, this new model was used for calculating the conditions in which transformation from stable to instable flow characteristics occur. It has been discovered that the flow becomes unstable at a pressure about $30 \%$ higher than bubble point pressure, which causes pulsation effect in the pipeline structure. However, homogenic behaviour should be observed in this hydrodynamic condition. Also, the model provides a guideline how to optimize the flow rate by adjusting the pipeline parameters to minimize the flow resistance, liquid slugging and hydraulic hammering effects which cause instable operation.

Acknowledgement: This study was sponsored by the Azerbaijan State Oil and Industrial University,“Gas, Petroleum and Mining” Faculty. We recognize the support of Scientific- Research Institute "Geotechnological problems of oil, gas and chemistry" and Institute of "Oil and Gas", Azerbaijan National Academy of Sciences. Also, the authors with to thank Robin Pringle (Operations Support) for his review and valuable advices. 


\section{References}

[1] Abbasov Z.Y., Fataliyev, V.M., The effect of gas-condensate reservoir depletion stages on gas injection and the aerosol state of fluids in this process, J. Nat. Gas Sci. Eng., 2016, 31, 779-790.

[2] Ali S.F., Yeung H., Experimental Investigation and Numerical Simulation of Two-Phase Flow in a Large-Diameter Horizontal Flow Line Vertical Riser, Petrol. Sci. Techn., 2010, 28(11), 1079-1095.

[3] Aliyu M.A., Yahaya D.B., Liyun L., Hoi Y., Kyung C.K., Interfacial friction in upward annular gas-liquid two-phase flow in pipes, Exp. Therm. Fluid Sci., 2017, 84, 90-109.

[4] Archibong-Eso A., Yan W., Baba Y., Kanshio S., Yeung H.,Viscous Liquid-Gas Flow in Horizontal Pipelines: Experiments and Multiphase Flow Simulator Assessment. BHR Group, BHR-2015-F3, 17th International Conference on Multiphase Production Technology, Cannes, France, 10-12 June 2015.

[5] Bordalo S.N., Morooka C.K., Slug flow induced oscillations on subsea petroleum pipelines, J. Petrol. Sci. Eng., 2018, 165, 535 549.

[6] Dabirian R., Mohan R., Shoham O., Kouba G., Critical sand deposition velocity for gas-liquid stratified flow in horizontal pipes, J. Nat. Gas Sci. Eng., 2016, 33, 527-537.

[7] Dall'Acqua D., Benucci M., Corvaro F., Leporini M., Grifoni R. C., Del Monaco A., ... and Marchetti B., Experimental results of pipeline dewatering through surfactant injection, J. Petrol. Sci. Eng., 2017, 159, 542-552.

[8] DuanJ., Liu H., Wang N., Gong J., Guangwei J., Hydro dynamic modeling of stratified smooth two-phase turbulent flow with curved interface through circular pipe, Int. J. Heat Mass Transf., 2015, 89, 1034-1043.

[9] Gato L.M.C., Henriques J.C.C., Dynamic behavior of high-pressure natural-gas flow in pipelines, Int. J. Heat Fluid Flow, 2005, 26(5), 817-825.

[10] Gazarez-Candia O., Espinoza-Paredes G., Numerical Study of Stratified Gas-Liquid Flow, Petrol. Sci. Technol., 2008, 26(1), 6476.

[11] Griffith P., Multiphase Flow in Pipes, SPE, J. Petrol. Technol., 1984, 36(03), 361-367.

[12] Hamidov N.N., Fataliyev V.M., Experimental study into the effectiveness of the partial gas cycling process in the gas-condensate reservoir development, Petrol. Sci. Technol., 2016, 34(7), 677684.

[13] Ismayilov G.G., Some ways for improvement the flow hydraulic of gas-liquid systems in a pipe with the presence of phase transitions, Scholarly notes, Azerbaijan State Oil Acad., 1997, 2, 206211, (In Russian).

[14] Jia W., Wu X., Li Ch., He Y., Characteristic analysis of a nonequilibrium thermodynamic two-fluid model for natural gas liquid pipe flow, J. Nat. Gas Sci. Eng., 2017, 40, 132-140.
[15] Kadri U., Mudde R.F., Oliemans R.V.A., Influence of the operation pressure on slug length in near horizontal gas-liquid pipe flow, Int. J. Multiphase Flow, 2010, 36(5), 423-431.

[16] Lebedev I.K., Hydrodynamics of steam boilers, Energoatomizdat, Moscow, 1987, (In Russian).

[17] Leporini M., Marchetti B., Corvaro F., Di Giovine G., Polonara F., Terenzi A., Sand transport in multiphase flow mixtures in a horizontal pipeline: An experimental investigation, Petrol., 2018, 1-10.

[18] Mirzadzhanzade A.Kh., Kuznetsov O.L., Basniev K.S., Aliev Z.S., Foundation of gas recovery technology, Nedra, Moscow, 2003, (In Russian),

[19] Nobakht Hassanlouei R., Firouzfar H., Kasiri N., Khanof M.H., A simple mathematical model for slug liquid holdup in horizontal pipes, Scientia Iranica, 2012, 19(6), 1653-1660.

[20] Pedersen S., Durdevic P.,Yang Z., Challenges in slug modeling and control for offshore oil and gas productions, Int. J. Multiphase Flow, 2017, 88, 270-284.

[21] Richard M.P., Marilyn E.K., Applied Colloid and Surface Chemistry, 2004, John Wiley \& Sons, Ltd.

[22] Sattarov R.M., Ismayilov G.G., Nabil I.A., The flow of gas-liquid systems in the regions of phase transformation, Scholarly notes, Azerbaijan State Oil Acad., 1997, 3, 224-230.

[23] Schubring D., Shedd T.A., A model for pressure loss, film thickness, and entrained fraction for gas-liquid annular flow, Int. J. Heat Fluid Flow, 2011, 32(3), 730-739.

[24] Suleymanov B.A., Abbasov E.M., Sisenbayeva M.R., Mechanism of Live Oil Viscosity Anomaly Near to Bubble Point Pressure, SOCAR Proceedings, 2017, 01, 35-45.

[25] Talaie M.R., Soleimani Deilamani K., A New Approach for Modeling of Gas-condensate Flow Through Pipelines under Industrial Operating Condition, J. Nat. Gas Sci. Eng., 2014, 21, 540-555.

[26] Usman M.A., Olatunde A.O., Akinlemibola O.J.,Modification of Pipeline Equations and Simulation of Transients in Compressible Fluid Flow in Pipes, Petrol. Sci. Technol., 2011, 29(18), 1963-1974.

[27] Wang L., Yang Y., Li Y., Wang Y., Resonance analyses of a pipelineriser system conveying gas-liquid two-phase flow with flowpattern evolution, Int. J. Press. Vess. Piping, 2018, 161, 22-32.

[28] Zhang J., Kang J., Fan J., Gao J., Study on erosion wear of fracturing pipeline under the action of multiphase flow in oil \& gas industry, J. Nat. Gas Sci. Eng., 2016, 32, 334-346.

[29] Zhang J., Kang J., Fan J., Gao J., Research on erosion wear of highpressure pipes during hydraulic fracturing slurry flow, J. Loss Prev. Proc. Industr., 2016, 43, 438-448.

[30] Zhao Y., Lao L., Yeung H., Investigation and prediction of slug flow characteristics in highly viscous liquid and gas flows in horizontal pipes, Chem. Eng. Res. Design, 2015, 102, 124-137. 